\title{
Correction to: Aging with multiple sclerosis: prevalence and profile of cognitive impairment
}

\author{
Mariana Branco ${ }^{1,2}$. Luis Ruano ${ }^{1,2,3}$. Emilio Portaccio ${ }^{4}$ - Benedetta Goretti ${ }^{4,5}$ - Claudia Niccolai $^{13}$. Francesco Patti $^{6}$. \\ Clara Chisari $^{6}$ • Paolo Gallo ${ }^{7}$ - Paola Grossi ${ }^{7,8}$. Angelo Ghezzi ${ }^{9}$. Marco Roscio ${ }^{9}$ Flavia Mattioli $^{10}$ • Fabio Bellomi ${ }^{10}$. \\ Marta Simone $^{11}$ - Rosa Gemma Viterbo ${ }^{12}$ - Maria Pia Amato ${ }^{5,13}$
}

Published online: 12 November 2019

(C) Fondazione Società Italiana di Neurologia 2019

\section{Correction to: Neurological Sciences (2019) 40:1651-1657 https://doi.org/10.1007/s10072-019-03875-7}

The above article was published online with an error in author name's affiliation. The Author Claudia Niccolai has changed her affiliation to IRCCS Fondazione Don Carlo Gnocchi, Florence, Italy.

The online version of the original article can be found at https://doi.org/ 10.1007/s10072-019-03875-7

Maria Pia Amato

mariapia.amato@unifi.it

1 Neurology Department, Centro Hospitalar de Entre Douro e Vouga, Santa Maria da Feira, Portugal

2 Departamento de Epidemiologia Clínica Medicina Preditiva e Saúde Pública, Faculdade de Medicina da Universidade do Porto, Porto, Portugal

3 EPIUnit, Instituto de Saúde Pública, Universidade do Porto, Porto, Portugal

4 SOC Neurologia - Firenze, AUSL Toscana Centro, Florence, Italy

5 Department NEUROFARBA, Section Neurosciences, University of Florence, Largo Brambilla 3, 50134 Florence, Italy
6 University of Catania, Catania, Italy

7 University of Padova, Padova, Italy

8 Department, ASST Crema, Neuroimmunology Center, Cardiocerebrovascular, Crema, Italy

9 Gallarate Hospital, Varese, Italy

10 ASST Spedali Civili Brescia Neuropsychology Unit, Brescia, Italy

11 Department of Basic Medical Sciences, Child and Adolescence Neuropsychiatry Unit, Neuroscience and Sense Organs University "Aldo Moro" Bari, Bari, Italy

12 University of Bari, Bari, Italy

13 IRCCS Fondazione Don Carlo Gnocchi, Florence, Italy 